\title{
Minimization of the shadow patterns produced by periodic mesh grids in extreme ultraviolet telescopes
}

\author{
Frédéric Auchère, ${ }^{1, *}$ Julien Rizzi, ${ }^{1}$ Anne Philippon, ${ }^{1}$ and Pierre Rochus ${ }^{2}$ \\ ${ }^{1}$ Institut d'Astrophysique Spatiale, CNRS/Université Paris-Sud 11, Bâtiment 121, 91405 Orsay, France \\ ${ }^{2}$ Centre Spatial de Liège, Université de Liège, Av. du Pré-Aily, 4031 Angleur, Belgium \\ ${ }^{*}$ Corresponding author: frederic.auchere@ias.u-psud.fr
}

Received October 20, 2010; accepted November 8, 2010;

posted November 15, 2010 (Doc. ID 136881); published December 22, 2010

\begin{abstract}
Thin metallic films are used as passband filters in space telescopes operating in the extreme ultraviolet (EUV). Because of their thinness, typically 100 to $200 \mathrm{~nm}$, they are very sensitive to static pressure differentials and to mechanic and acoustic vibrations. Therefore, they are difficult to manage in all phases of a space program, from manufacturing to vacuum testing to launch. A common solution to this problem is to reinforce them with fine mesh grids with pitches ranging from a few hundred micrometers to a few millimeters. Depending on their location in the optical path, the main effect of these periodic grids is either to diffract light or to cast penumbral shadows on the focal plane. In this paper, we analyze the formation of the shadow modulation patterns and derive design rules to minimize their amplitude. The minimization principle is illustrated by an application to a solar EUV telescope. (C) 2010 Optical Society of America OCIS codes: $\quad 080.0080,080.2740,220.4830,310.0310$
\end{abstract}

\section{INTRODUCTION}

In solar extreme ultraviolet (EUV) telescopes, thin-film metallic filters are used to reject the infrared and visible part of the solar spectrum. Two filters are generally used. The first one, at the entrance of the instrument, rejects most of the heat and the visible light. The second filter, located somewhere down the optical path, provides additional rejection and redundancy to protect against pinholes in the front filter. These membranes (typically $150 \mathrm{~nm}$ thick) are usually reinforced either by a metallic mesh grid, a thin plastic foil (e.g., polyimide), or a combination of both. Mesh grids can be a few tens of micrometers thick with a pitch ranging from a few tenths of a millimeter to a few millimeters. The generic configuration of these instruments is illustrated in Fig. 1(a).

The optical effects of these grids depend on their location in the optical path. Being located in a parallel beam, the first grid has a diffracting effect. The second grid is typically positioned close to the focal plane in a converging beam. Its diffraction is generally negligible because its distance to the focal plane is short, but it casts a periodic shadow pattern. Figure 1(b) illustrates the basic principle of the formation of the grid modulation. It results from the periodic blocking of part of the beam by the grid rods.

Figure 2 shows the effect in the raw images obtained by the EUV imaging telescope (EIT)[1] on board the SOHO spacecraft. Similar patterns are observed in the images obtained by the EUV imagers on board STEREO [2] or the atmospheric imaging assembly on board SDO. As discussed by [3], the 15\% periodic modulation can be filtered out of the EIT images using Fourier transform-based techniques. However, such processing creates inevitable artifacts because some of the power at the filtered frequencies is of solar origin. Theoretical modeling of the EIT grid, including diffraction effects, was also performed [4]. Because this method supposes knowledge of the instrument geometry within an accuracy that is not easily achievable, the results are not directly applicable to correct data. If possible, the grid pattern modulation can be calibrated on the ground, but the corresponding correction matrix is necessarily affected by uncertainties and noise.

None of the aforementioned methods allow a completely satisfactory removal of the grid from the images. Indeed, a residual pattern can still be detected in fully calibrated EIT data. The most robust approach would be to cancel the modulation by design of the telescopes, eliminating the need for subsequent image processing. In this paper, we investigate the properties of the grid patterns in these instruments as a function of their geometrical properties. We demonstrate that the grid pattern can be minimized or suppressed in certain configurations.

\section{MODULATION AND MINIMIZATION}

\section{A. Principle}

The amount $T$ of light geometrically transmitted by the grid can be expressed as the convolution between the grid transmission curve and the beam cross section

$$
T(\mu, \nu)=\int_{-a / 2}^{a / 2} \int_{-b / 2}^{b / 2} G(x, y) B(x-\mu, y-\nu) \mathrm{d} x \mathrm{~d} y
$$

where $G$ is the grid transmission, $B$ is the beam cross section, $a$ and $b$ are the total width and height of the grid aperture, respectively, and $\mu$ and $\nu$ are the positions of the beam relative to the grid. The variations of the shape of the beam across the field of view (FOV) are supposed to be negligible. 


$\begin{gathered}\text { Entrance Filter } \\ \text { \& Grid }\end{gathered}$
Focal Filter
\& Grid

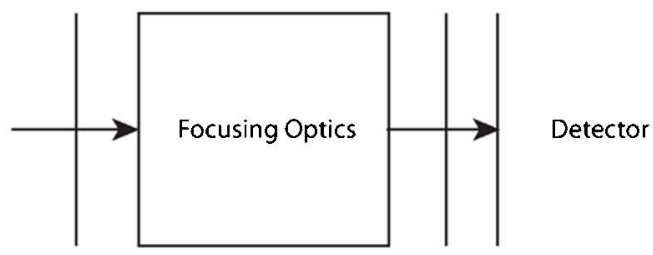

(a)

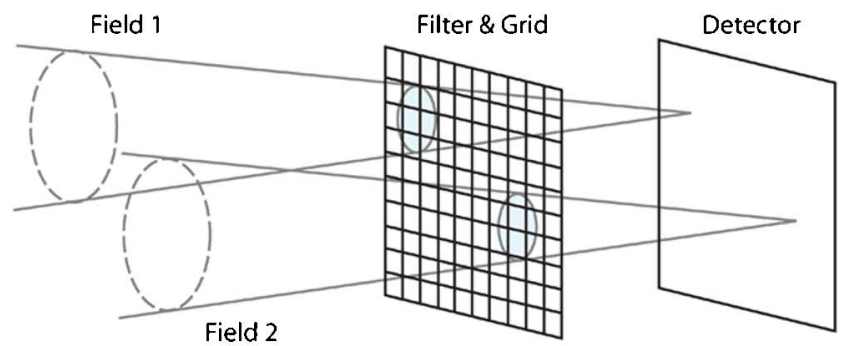

Filter \& Grid

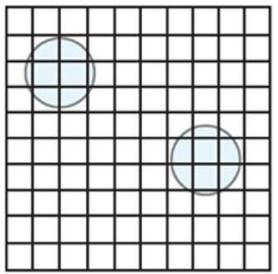

(b)

Fig. 1. (a) Generic configuration of the instruments considered in this paper. (b) Principle of the grid pattern modulation. The periodic filter support grid masks different fractions of the converging beam for different fields, resulting in a periodic modulation of the signal across the detector.

The variations of $T(\mu, \nu)$ can be minimized in specific geometric configurations. The principle of the minimization of the modulation is illustrated in one dimension in Fig. 3 . The dashed square curve represents the grid transmission. The solid top hat represents the beam cross section at the filter plane. In the left column, the beam moves from left to right across the field. The amount of transmitted light (the shaded surface below the solid and dashed curves) increases from the top to the middle configuration, but remains constant from the middle to the bottom configurations. This implies a variation of the transmission and, therefore, a modulation of the signal

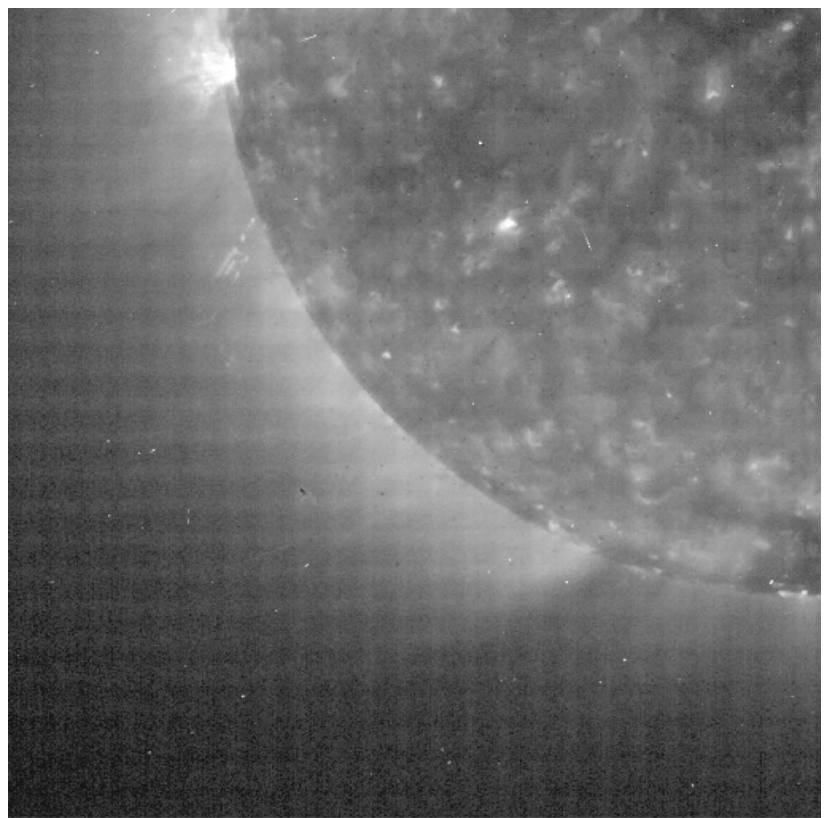

Fig. 2. Periodic modulation in raw EIT images produced by the focal plane filter grid. across the FOV. In the right column, the beam width is equal to two grid periods. In this case, as the beam moves from left to right, an extra amount of light transmitted or blocked at one edge of the beam is exactly compensated at the other end. Therefore, the total amount of transmitted light is independent from the beam position, resulting in a zero modulation. This holds true as long as the beam width is equal to an integer number of grid periods.

This principle can be generalized in two dimensions on the condition that the grid pattern and the beam have the same shape. The two possible solutions are the square and the hexagon (Fig. 4).

\section{B. Simulations}

To verify this principle, we modeled a practical case: the full sun imager (FSI) on board the Solar Orbiter mission. FSI was first described in [5]. It is designed to observe the Sun in two narrow bands of the EUV spectrum centered on the $17.4 \mathrm{~nm}$ line of $\mathrm{Fe} \mathrm{X}$ and on the $30.4 \mathrm{~nm}$ line of He II. The general layout of the instrument is given in Fig. $\underline{5}$. A $5 \mathrm{~mm}$ diameter pupil is located $700 \mathrm{~mm}$ in front of an elliptical mirror. A thin-film aluminum filter is located $310 \mathrm{~mm}$ behind the pupil. Two types of thin-film filters (zirconium and magnesium) are held by a filter wheel located close to the $3 \mathrm{k} \times 3 \mathrm{k}$ detector. The two types of filters isolate one of the two narrow bands reflected by the mirror's dual-band multilayer coating [6] . The support grids are nominally 70 lines per inch meshes with $81 \%$ geometrical transmission.

Considering the proximity of the focal filter from the focal plane, diffraction is a second-order effect and was neglected. The simulations were made using Interactive Data Language. All the geometrical parameters being known, we computed for each pixel of the detector the corresponding position of the center of the beam (chief ray) on the filter. For each pixel, we then built a binary matrix representing the multiplication 

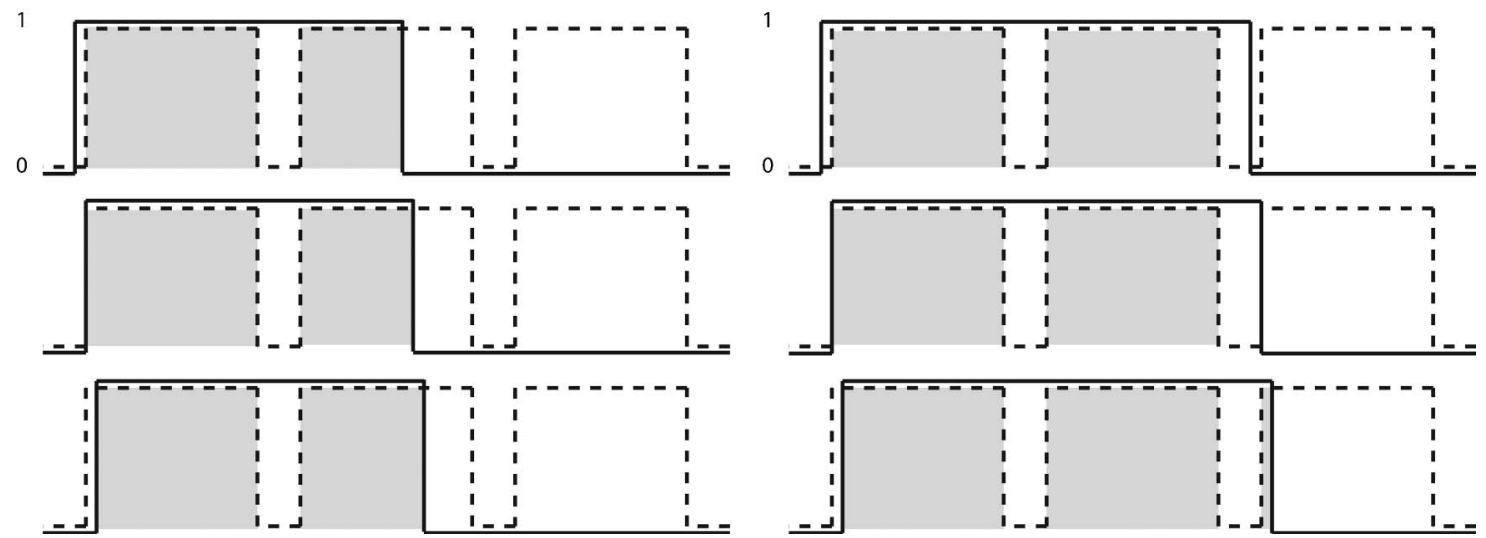

Fig. 3. One-dimensional illustration of the principle of the minimization of the grid modulation. The dashed square curve represents the grid transmission, the solid top hat represents the beam cross section at the filter, and the shaded areas represent the amount of transmitted light. Left column, an ordinary beam width results in a nonzero modulation when the beam moves from left to right (top to bottom panels); right column, the modulation cancels out for a beam width equal to an integer number of grid periods (two in this example). See Section 2 for details.

of the grid transmission by a top hat beam profile. The sum of this matrix normalized to the area of the unobstructed beam gives the effective transmission of the grid for the corresponding position in the FOV.

From the argument of Section 2 , we predicted that minima of the grid pattern modulation can be obtained for specific values of the size of the beam relative to the pitch of the grid. In the square or hexagonal cases, the modulation should cancel if the beam width is an integer number of the grid pitch. The size of the beam relative to the grid can be modified either by translating the filter along the beam at constant pitch, changing the grid pitch at constant position, or a combination of both. We chose to keep the nominal geometry of the instrument fixed and to vary the grid pitch. In all cases, we kept the width of the grid rods at $10 \%$ that of the grid pitch, so that the geometric transmission of the grids was always $81 \%$. The amount of modulation is estimated by the relative peak-tovalley variation and the standard deviation of the central region $(200 \times 200$ pixels $)$ of the computed grid patterns.

\section{RESULTS}

\section{A. Nominal Geometry}

We first ran simulations in the nominal geometry, i.e., with a square support grid and a circular beam. In this condition, the beam is $1.38 \mathrm{~mm}$ wide at the filter, which represents 3.8 periods of the nominal 70 lines per inch grid. The geometry of the grid is defined in Fig. 4 . The center image in the top panel of Fig. 6 shows, for the central $200 \times 200$ detector pixels, the modulation pattern obtained with such a standard grid. The left and right images are the patterns corresponding to the closest pitches that produce minimum $(326 \mu \mathrm{m})$ and maximum (371 $\mu \mathrm{m})$ modulation, respectively. To ease comparison, the same gray scale is used in the three images. The bottom panel in Fig. 6 shows the relative peak-to-valley variation (dashed curve, left scale) and the standard deviation (solid curve, right scale) of the computed patterns as a function of grid pitch (top scale) and the number of grid periods covered by the beam (bottom scale). On average, the amount of modulation decreases as the beam width increases compared to the grid pitch. This is consistent with the rule of thumb that focal
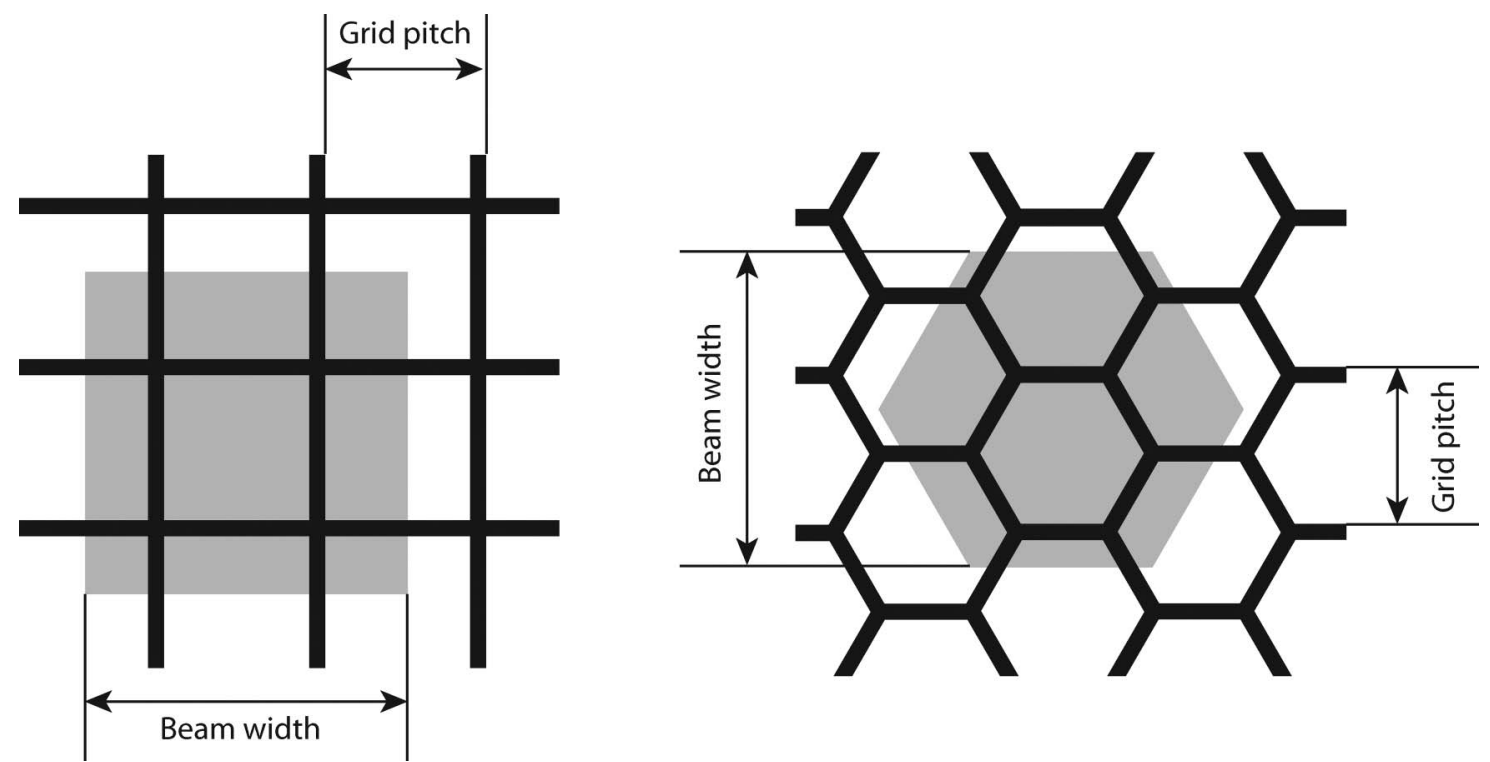

Fig. 4. Two possible geometries for optimal grid pattern minimization. Left, square grid pattern (black) and beam (gray); right, hexagonal grid pattern (black) and beam (gray). 


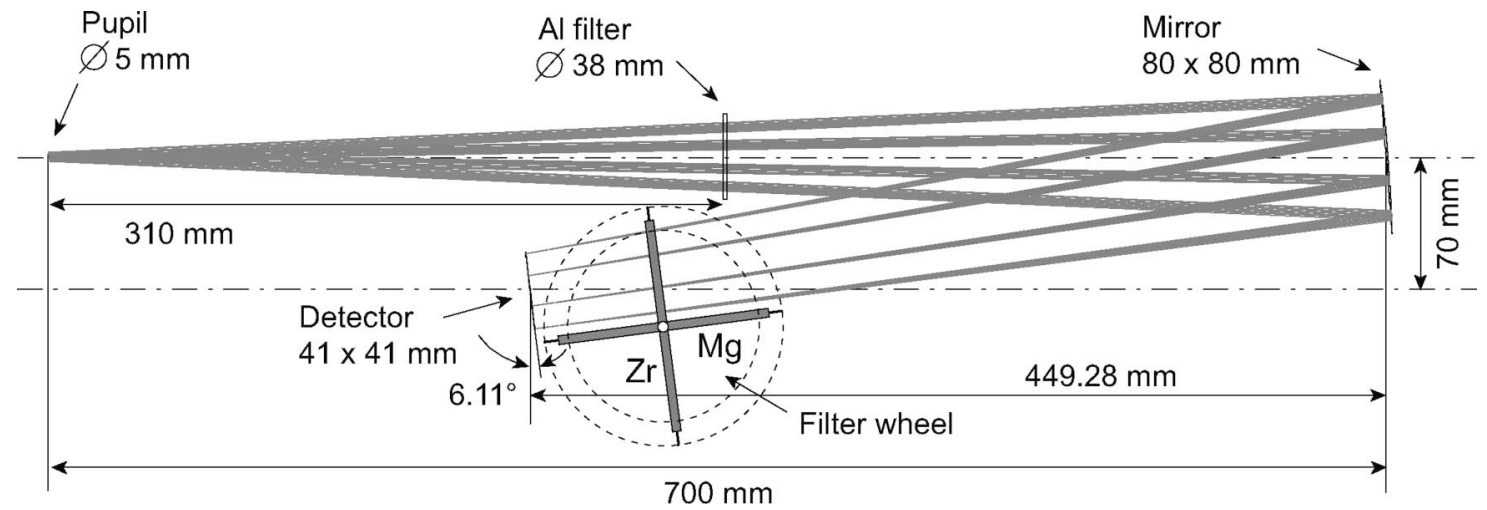

Fig. 5. Optical layout of the FSI.

filters should be located far from the detector in order for the penumbral shadow to be as diluted as possible. We find the modulation reaches local minima as we vary the ratio between beam width and grid pitch, even if the support grid and the beam do not have the same geometry. However, these minima are not zeroes. As expected, the modulation is a periodic function of the number of grid periods covered by the beam, but, in this case of a circular pupil and a square grid, the minima do not correspond to integer numbers of periods. With a pitch of $361 \mu \mathrm{m}$, the nominal grid produces a modulation close to a local maximum. Changing the grid pitch to $326 \mu \mathrm{m}$ reduces the amount of modulation by about a factor of 2 .
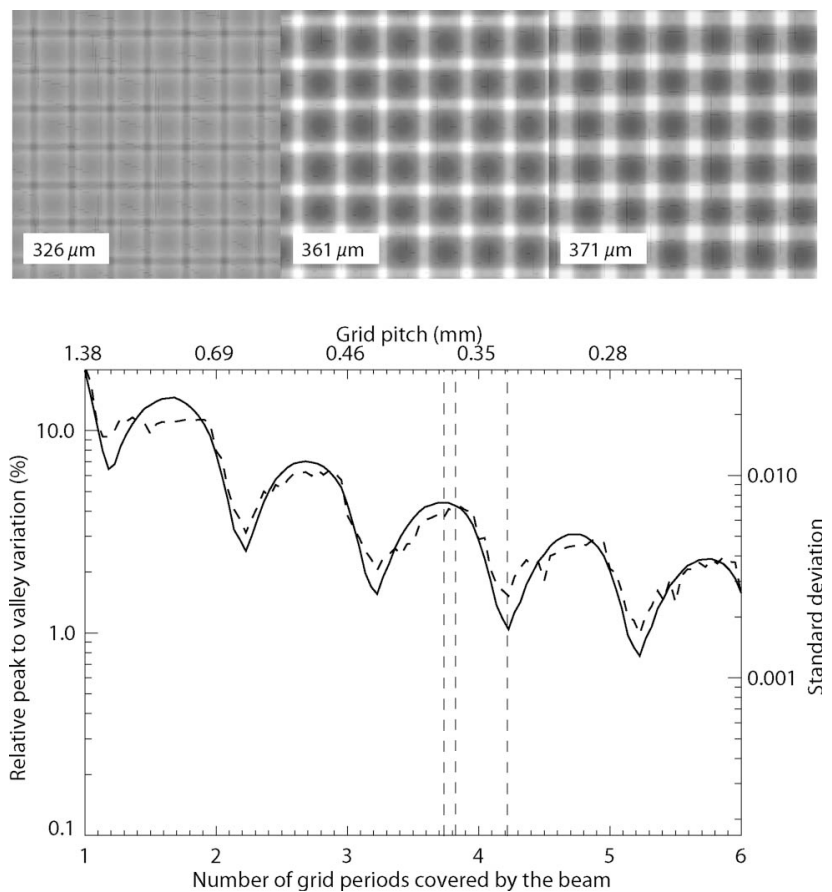

Fig. 6 . Top, central part $(200 \times 200$ pixels $)$ of the simulated modulation patterns in the nominal geometry of the FSI for three grid pitches. The center image corresponds to a pitch similar to that of the standard 70 lines per inch Luxel grid $(361 \mu \mathrm{m})$. The left and right images are the patterns corresponding to the closest pitches that produce minimum $(326 \mu \mathrm{m})$ and maximum $(371 \mu \mathrm{m})$ modulation, respectively. To ease comparison, the same gray scale is used for all images. Bottom, modulation of the shadow pattern (standard deviation, solid curve and right scale; peak-to valley relative variation, dashed line and left scale) as a function of the grid pitch (top scale) and number of grid periods covered by the beam (bottom scale) in the nominal FSI geometry (square grid and circular beam). The vertical dashed lines correspond to the three pitches of the top panel.

\section{B. Square and Hexagonal Geometries}

We performed the same aforementioned simulations after changing the pupil to a square and a hexagon in order to obtain a square and hexagonal beam at the filter. To keep the photon flux constant, we considered square and hexagonal pupils having the same surface as the nominal $5 \mathrm{~mm}$ diameter circular pupil. We have

$$
\begin{gathered}
A_{\text {circle }}=\pi r_{\text {circle }}^{2} \\
A_{\text {square }}=r_{\text {square }}^{2}, \\
A_{\text {hexagon }}=\frac{3 \sqrt{3}}{2} r_{\text {hexagon }}^{2},
\end{gathered}
$$

where $A_{x}$ and $r_{x}$ are, respectively, the area and radius (for the circle) or edge (for the square and the hexagon) of the pupil in the three geometries. Equating the area of the square and the hexagon to that of the circle, we find
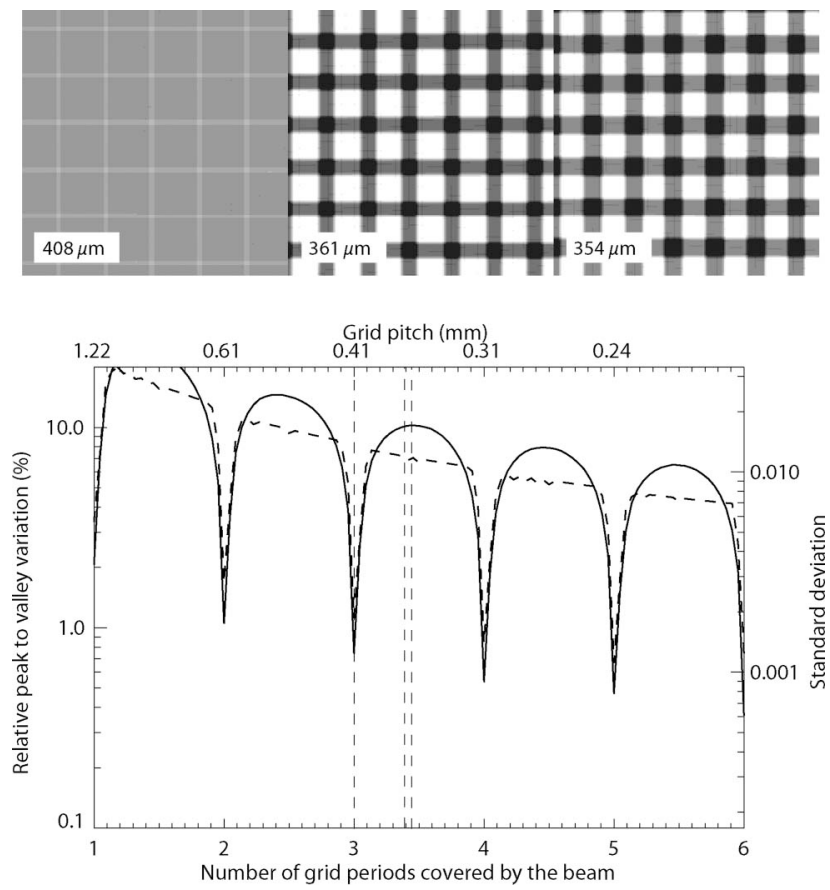

Fig. 7. Same as Fig. 6 in the case of a square beam with a square grid. The center picture corresponds to the nominal grid pitch $(361 \mu \mathrm{m})$. The left and right images are the patterns that correspond to the closest pitches that produce minimum $(408 \mu \mathrm{m})$ and maximum $(354 \mu \mathrm{m})$ modulation, respectively. The gray scale is the same as in Fig. 6. 


$$
\begin{gathered}
r_{\text {square }}=r_{\text {circle }} \sqrt{\pi} \approx 1.772 r_{\text {circle }}, \\
r_{\text {hexagon }}=r_{\text {circle }} \sqrt{\frac{2 \pi}{3 \sqrt{3}}} \approx 1.099 r_{\text {circle }} .
\end{gathered}
$$

The geometries of the square and hexagonal grids and beams are illustrated in Fig. $\underline{4}$. As before, the grid thickness is always adjusted to keep a constant geometrical transmission of $81 \%$. The top panel in Fig. 7 shows, for the square case, the computed grid patterns for the nominal pitch $(361 \mu \mathrm{m}$, center image) and for the closest pitches that produce minimum (408 $\mu \mathrm{m}$, left image) and maximum (354 $\mu \mathrm{m}$, right image) modulation. The gray scale is the same as in Fig. 6 . The bottom panel shows the amount of modulation as a function of grid pitch (top scale) and number of grid periods covered by the beam (bottom scale). We obtain lower minima in this geometry than in the nominal case (circular pupil and square grid), but also higher maxima. Again, the nominal grid pitch produces a modulation close to a local maximum.

Figure $\underline{8}$ shows the same information in the hexagonal case. We now obtain lower minima and also lower maxima compared to the circular pupil and square grid case. We also note additional secondary minima for half numbers of grid periods covered by the beam.

\section{Grid Pattern Cancellation}

Figure 9 compares the results of the three cases described earlier. The square pupil and grid case produces deeper minima than the nominal case, but also higher maxima, while the hexagonal pupil and grid case produces lower minima and maxima. The amount of modulation depends on the geometry of the pupil and the grid. Minima are obtained in certain config-
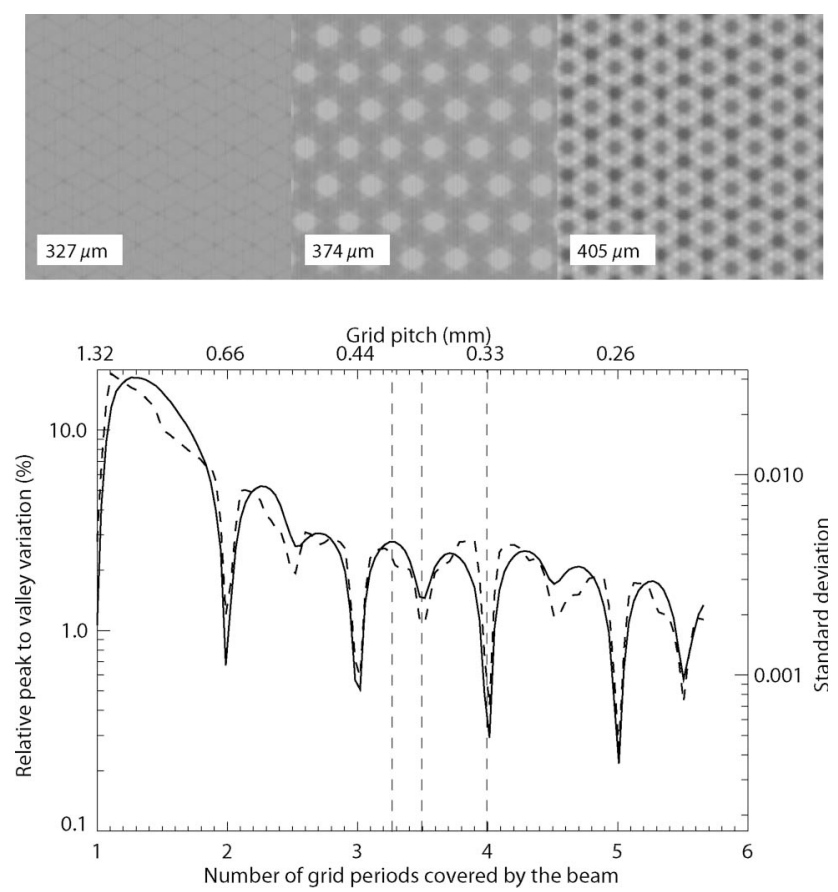

Fig. 8. Same as Fig. 6 in the case of a hexagonal beam and grid. The center picture corresponds to a pitch of $374 \mu \mathrm{m}$, producing intermediate modulation. The left and right images are the patterns corresponding to the closest pitches that produce minimum $(327 \mu \mathrm{m})$ and maximum $(405 \mu \mathrm{m})$ modulation, respectively. The gray scale is the same as in Fig. $\underline{6}$.

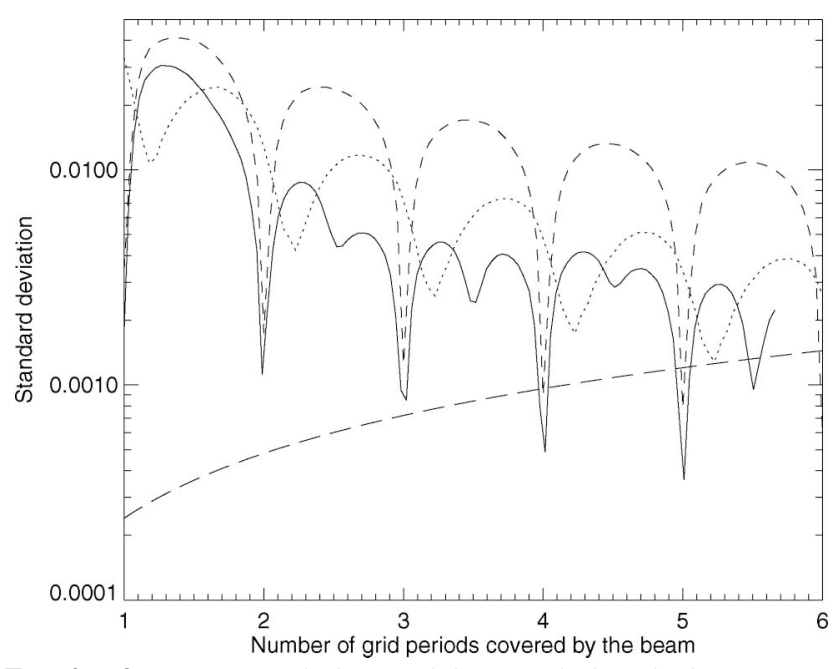

Fig. 9. Comparison of the modulation of the shadow pattern (standard deviation) in the three cases of Fig. $\underline{6}$ (dotted curve, circular pupil, and square grid), Fig. 7 (dashed curve, square pupil and grid), and Fig. 8 (solid curve, hexagonal pupil and grid) as a function of the number of grid periods covered by the beam. The long-dashed curve represents the modulation level below which the grid pattern is practically undetectable. See Subsection 3.C for details.

urations (but not zeroes). This is because the width of the beam at the filter is not exactly constant across the FOV. However, we will show that the modulation level is low enough in certain configurations to be practically undetectable considering the local noise level in the images.

Assuming perfect charge collection, the signal recorded by a CCD detector can be expressed as

$$
S=N \frac{\eta_{i}}{G}
$$

where $N$ is the number of interacting photons, $\eta_{i}$ is the quantum yield, and $G$ is the analog-to-digital converter gain. The number of interacting photons has a variance

$$
\sigma_{N}^{2}=N+N^{2} \sigma_{G}^{2}
$$

where $\sigma_{G}^{2}$ is the variance of the grid pattern as computed in Section 3 . The first term corresponds to the photon shot noise, and the second term corresponds to the grid modulation. Neglecting variations of the quantum yield and the gain, the variance of the detected signal can then be written as

$$
\sigma_{S}^{2}=\left(\frac{\partial S}{\partial N}\right)^{2} \sigma_{N}^{2}+\sigma_{R}^{2}=\frac{\eta_{i}^{2}}{G^{2}} N+\frac{\eta_{i}^{2}}{G^{2}} N^{2} \sigma_{G}^{2}+\sigma_{R}^{2},
$$

where $\sigma_{R}^{2}$ is the variance of the detector read noise, from which we derive that the grid pattern is practically undetectable if

$$
\sigma_{G}^{2}<\frac{1}{N}+\left(\frac{G}{N \eta_{i}}\right)^{2} \sigma_{R}^{2} .
$$

This relation shows that the grid is most easily detected in the case of low noise and high signal. This effect in clearly seen in Fig. 2. The number of photons $N$ to be considered is not that collected in a single pixel but that integrated over $n^{2}$ pixel, $n$ being a half grid period. Indeed, if one wants to detect the grid modulation in an image, it is possible to rebin 
the image to increase the signal-to-noise ratio. Rebinning more than a half grid pitch would completely suppress the grid.

For our application, we assumed a worst case of zero read noise and exposure times adjusted to produce, on average, 1000 detected photons per pixel. In this case, the relation 10 simplifies to $\sigma_{G}^{2}<1 / N$. The corresponding maximum values of $\sigma_{G}$ as a function of the grid pitch (and therefore $n$ ) are represented by the long-dashed curve in Fig. 9 .

In the nominal case of a circular pupil combined with a square grid, the standard deviation of the modulation pattern is never lower than the dashed curve in the range of grid pitches considered (down to $180 \mu \mathrm{m}$ ), which means that the grid can always be detected. Considering the trend visible in Fig. 9, the grid would, however, become undetectable in this configuration with finer grid pitches or (which is equivalent) more grid periods covered by the beam. In the square pupil and square grid configuration, the grid becomes undetectable for a few grid pitches around $240 \mu \mathrm{m}$. The hexagonal pupil and hexagonal grid configuration is the most favorable, with a cancellation of the grid for pitches around 330 and $260 \mu \mathrm{m}$.

\section{CONCLUSIONS}

The support grids of the thin-film filters used in EUV telescopes produce periodic penumbral shadow patterns on the focal planes of the instruments. This modulation results from the convolution of the beam profile at the filter by the periodic grid transmission. The usual rule of thumb for minimizing this effect is to position the filter far from the detector so as to dilute the penumbral shadow as much as possible. We have shown that the amplitude of the modulation pattern can, in fact, be minimized by properly choosing the geometries of the grid and/or entrance pupil. We showed that if pupil and grid pattern both have either a square or hexagonal geometry, the amplitude of the modulation is at a minimum when the beam covers integer numbers of grid pitches.

We investigated in detail the practical case of the FSI on board the Solar Orbiter mission. These simulations showed that the nominal configuration was not optimal. The amount of modulation was found to be a pseudoperiodic function of the ratio between the beam width at the filter and the grid pitch. In the nominal case (square grid and circular pupil), the amplitude of the modulation can be reduced by about a factor of 2 by adjusting the grid pitch from 361 to $326 \mu \mathrm{m}$. The modulation can be further minimized if the support grid and the entrance pupil are both either square or hexagonal. Since modifying the shape of the pupil while keeping its surface unchanged has no impact on the optical performances, our results show that the hexagonal pupil and grid geometry is the most favorable configuration for this instrument. In this case, the modulation can be reduced sufficiently so that the grid pattern becomes practically invisible in the images considering the expected signal-to-noise ratio.

Since there is usually freedom in the choice of grid pitch and filter to detector distance, we suggest that they be chosen according to the aforementioned principles in future instruments. Similar simulations can be used to derive the optimum configuration.

\section{REFERENCES}

1. J.-P.Delaboudinière, G. E. Artzner, J. Brunaud, A. H. Gabriel, J.-F. Hochedez, F. Millier, X.-Y. Song, B. Au, K. P. Dere, R. A. Howard, R. Kreplin, D. J. Michels, J. D. Moses, J.-M. Defise, C. Jamar, P. Rochus, J.-P. Chauvineau, J.-P. Marioge, R. C. Catura, J. R. Lemen, L. Shing, R. A. Stern, J. B. Gurman, W. M. Neupert, A. Maucherat, F. Clette, P. Cugnon, and E. L. van Dessel, "EIT: extreme-ultraviolet imaging telescope for the SOHO mission," Sol. Phys. 162, 291-312 (1995). http://adsabs.harvard.edu/abs/1995SoPh..162..291D

2. R. A. Howard, J. D. Moses, A. Vourlidas, J. S. Newmark, D. G. Socker, S. P. Plunkett, C. M. Korendyke, J. W. Cook, A. Hurley, J. M. Davila, W. T. Thompson, O. C. St Cyr, E. Mentzell, K Mehalick, J. R. Lemen, J.-P. Wuelser, D. W. Duncan, T. D. Tarbell, C. J. Wolfson, A. Moore, R. A. Harrison, N. R. Waltham, J. Lang, C. J. Davis, C. J. Eyles, H. Mapson-Menard, G. M. Simnett, J.-P. Halain, J.-M. Defise, E. Mazy, P. Rochus, R. Mercier, M.-F. Ravet, F. Delmotte, F. Auchère, J.-P. Delaboudinière, V. Bothmer, W. Deutsch, D. Wang, N. Rich, S. Cooper, V. Stephens, G. Maahs, R. Baugh, D. McMullin, and T. Carter, "Sun earth connection coronal and heliospheric investigation (SECCHI)," Space Sci. Rev. 136, 67-115 (2008).

3. D. Moses, F. Clette, J.-P. Delaboudinière, G. E. Artzner, M. Bougnet, J. Brunaud, C. Carabetian, A. H. Gabriel, J. F. Hochedez, F. Millier, X.-Y. Song, B. Au, K. P. Dere, R. A. Howard, R. Kreplin, D. J. Michels, J. M. Defise, C. Jamar, P. Rochus, J. P. Chauvineau, J. P. Marioge, R. C. Catura, J. R. Lemen, L. Shing, R. A. Stern, J. B. Gurman, W. M. Neupert, J. Newmark, B. Thompson, A. Maucherat, F. Portier-Fozzani, D. Berghmans, P. Cugnon, E. L. van Dessel, and J. R. Gabryl, "EIT observations of the extreme ultraviolet sun,” Sol. Phys. 175, 571-599 (1997).

4. J.-M. Defise, "Analyse des performances instrumentales du télescope spatial EIT,” Ph.D. thesis, (Université de Liège, 2000).

5. F. Auchère, X.-Y. Song, F. Rouesnel, T. Appourchaux, J.-J. Fourmond, J.-C. Le Clec'h, M. Berthé, J.-M. Defise, E. Mazy, P. Rochus, R. Mercier, and M.-F. Ravet, "Innovative designs for the imaging suite on Solar Orbiter," Proc. SPIE 5901, 298-304 (2005).

6. C. Hecquet, F. Delmotte, M.-F. Ravet-Krill, S. de Rossi, A. Jérome, F. Bridou, F. Varnière, E. Meltchakov, F. Auchère, A. Giglia, N. Mahne, and S. Nanaronne, "Design and performance of two-channel EUV multilayer mirrors with enhanced spectral selectivity," Appl. Phys. A 95, 401-408 (2009). 